\title{
Defining Major Surgery: A Delphi Consensus Among European Surgical Association (ESA) Members
}

\author{
David Martin $^{1} \cdot$ Styliani Mantziari $^{1} \cdot$ Nicolas Demartines $^{1} \cdot$ Martin Hübner $^{1}$ on behalf of \\ the ESA Study Group
}

Published online: 14 March 2020

(c) Société Internationale de Chirurgie 2020

\begin{abstract}
Background Major surgery is a term frequently used but poorly defined. The aim of the present study was to reach a consensus in the definition of major surgery within a panel of expert surgeons from the European Surgical Association (ESA).

Methods A 3-round Delphi process was performed. All ESA members were invited to participate in the expert panel. In round 1, experts were inquired by open- and closed-ended questions on potential criteria to define major surgery. Results were analyzed and presented back anonymously to the panel within next rounds. Closed-ended questions in round 2 and 3 were either binary or statements to be rated on a Likert scale ranging from 1 (strong disagreement) to 5 (strong agreement). Participants were sent 3 reminders at 2 -week intervals for each round. $70 \%$ of agreement was considered to indicate consensus.

Results Out of 305 ESA members, 67 (22\%) answered all the 3 rounds. Significant comorbidities were the only preoperative factor retained to define major surgery (78\%). Vascular clampage or organ ischemia (92\%), high intraoperative blood loss (90\%), high noradrenalin requirements (77\%), long operative time (73\%) and perioperative blood transfusion (70\%) were procedure-related factors that reached consensus. Regarding postoperative factors, systemic inflammatory response $(76 \%)$ and the need for intensive or intermediate care $(88 \%)$ reached consensus. Consequences of major surgery were high morbidity ( $>30 \%$ overall) and mortality $(>2 \%)$.

Conclusion ESA experts defined major surgery according to extent and complexity of the procedure, its pathophysiological consequences and consecutive clinical outcomes.
\end{abstract}

David Martin and Styliani Mantziari shared first authorship.

Collaborators of the ESA Study Group are co-authors of this study and can be found under the heading Acknowledgements.

Electronic supplementary material The online version of this article (https://doi.org/10.1007/s00268-020-05476-4) contains supplementary material, which is available to authorized users.

Nicolas Demartines

demartines@chuv.ch

1 Department of Visceral Surgery, Lausanne University Hospital CHUV, University of Lausanne, Rue du Bugnon 46, 1011 Lausanne, Switzerland

\section{Introduction}

What constitutes major surgery has been a matter of debate since the beginning of surgical practice. As early as 1917, Dr. R. Earl was preoccupied by this lack of clear definition and its impact on the surgical profession, as at the time osteopaths were allowed to perform 'minor surgery' [1]. To his relevant inquiry, he received the following response: 'major surgery includes all work requiring a general anesthetic; all operations which involve openings into the great cavities of the body; all operations in the course of which hazards of severe hemorrhage are possible; all conditions in which the life of the patient is at stake; all 
conditions which require for their relief manipulations, for the proper performance of which special anatomical knowledge and manipulative skill are essential' [1]. In 1965, a broad-scale survey was conducted among American surgeons to define criteria for major surgery [2]. Twelve variables stood out, including the severity of surgical trauma, patient status, potential morbi-mortality and long-term sequelae, as well as the infrastructure and expertise needed. No other attempt to reach a consensual definition of major surgery has been published since.

Many healthcare providers argue to this day the existence of 'minor surgery,' as even a minor intervention may have serious side effects on some occasions. Nonetheless, 'major surgery' remains a term widely used, though poorly defined, which may have direct implications on healthcare management, adequate preoperative patient selection and preparation and even on residents' and surgeons' training [3-5]. Furthermore, this lack of standard definition, particularly in clinical trials, may confound the interpretation of surgical outcomes.

Generally, an expert is a person who, in addition to having a theoretical knowledge of a defined area, has acquired advanced practical competence and recognized by his peers in the field. These skills seemed interesting to us to establish a definition of major surgery. The European Surgical Association (ESA) was founded in 1993 and currently represents one of Europe's most prestigious surgical organizations, regrouping expert surgeons from leading medical institutions. The aim of the present study was to reach a consensus in the definition of major surgery within this panel of expert surgeons by means of a 3-round Delphi process.

\section{Materials and methods}

\section{Participants and study design}

ESA members include the continent's most prominent surgeons from the country's leading academic medical institutions, many of whom are Chairs of Surgery Department at these institutions. Membership also includes some leading surgeons from around the world.

All ESA members $(n=305)$ were invited to take part in a 3-round Delphi process, by means of electronic mail invitations and questionnaires delivered via a dedicated online platform (Survey Monkey ${ }^{\circledR}$, Palo Alto, CA, USA). The Delphi process involves completion of a series of questionnaires interspersed with summary and feedback derived from previous responses [6]. It allows free discussion without the influence of personal status, enables the alteration of personal views without embarrassment and provides a means to combine opinions from experts who are geographically dispersed [6-8]. This method has been widely used in surgery research.

In round 1, experts were presented with open- and closed-ended questions to define major surgery and determine its different aspects. Preoperative, intraoperative and postoperative parameters, as well as patient- and intervention-related criteria were considered. Significant comorbidities were defined as Charlson index $>3$ and/or ASA score $>2$ [9, 10]. The authors developed these parameters which seemed relevant to the perioperative phase, but all participants were asked to propose any additional criteria or reflexions they considered important in free-text fields. Results were analyzed and summarized in rounds 2 and 3, where only closed-ended questions were included, either binary (yes/no answer) or statements to be rated on a 5-scale Likert scale (1: strong disagreement, 5: strong agreement). The top 5 responses from the previous round were presented in return. For some pertinent factors, cutoffs have been proposed, and always with the possibility of choosing a proposed cutoff or proposing one in a freetext field. Participants were sent 3 reminders at 2 -week intervals for each round, with four-week intervals between rounds for result analysis and preparation of the next step. The whole process started on June 1, 2018, and was completed on February 1, 2019. All responses were treated anonymously, while participant's demographics were recorded. In accordance with previous studies, consensus was obtained when a statement was agreed or strongly agreed (Likert scale $\geq 4$ ), or a binary question answered 'yes' or 'no' by $\geq 70 \%$ of the experts $[11,12]$.

The study did not require approval by the local ethics committee as no patients were enrolled.

\section{Statistical analysis}

Descriptive statistics for categorical variables were reported as number and percentage, while continuous variables were reported as means and standard deviation (SD).

\section{Results}

Out of 305 ESA members, $67(22 \%)$ answered all the 3 rounds (Fig. 1). Participant demographics are shown in Table 1. Mean age was 59 years (SD 9), 100\% were males, $40 \%$ were specialized in HPB surgery, $75 \%$ had more than 20 years of practice and most of them practice in university hospitals (91\%). Among the experts, answering all three rounds, $40 \%$ were specialized in HPB surgery, $21 \%$ in upper gastrointestinal surgery and $18 \%$ colorectal surgery. A distinction between major and minor surgery was considered as important by $99 \%$ of ESA experts. There was no significant difference in demographics between the 
Fig. 1 Flowchart of experts' recruitment and participation
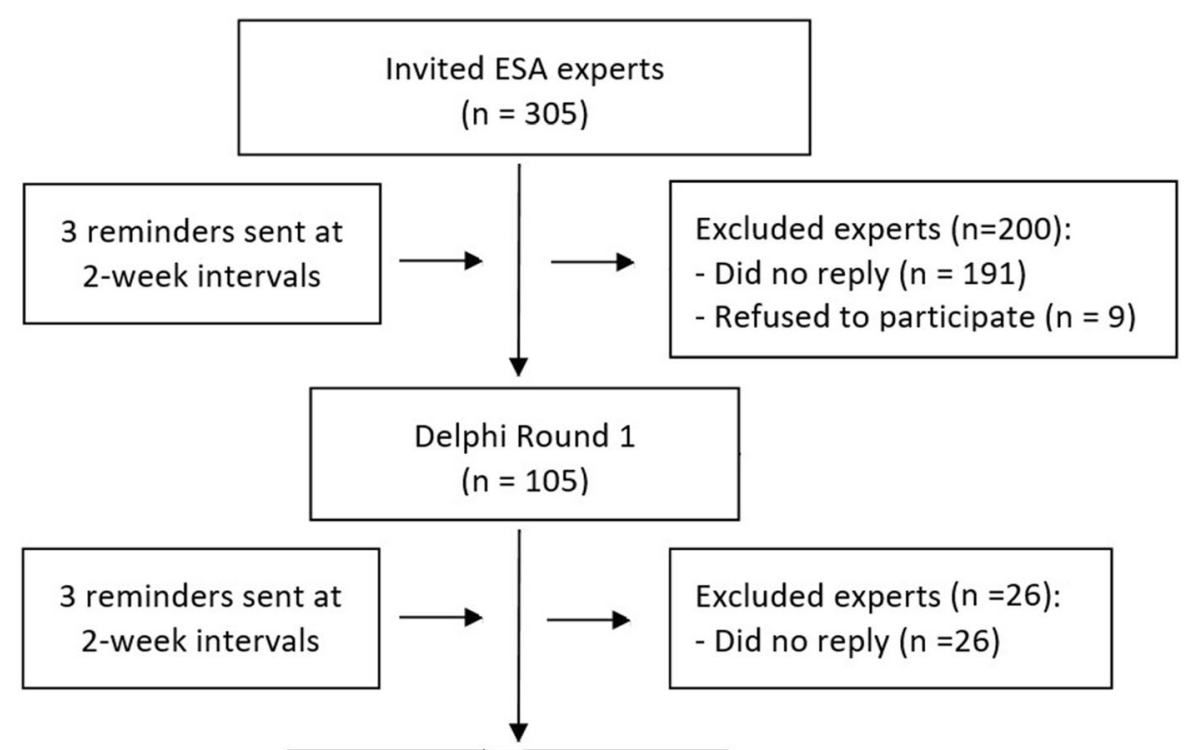

Delphi Round 2

$(n=79)$

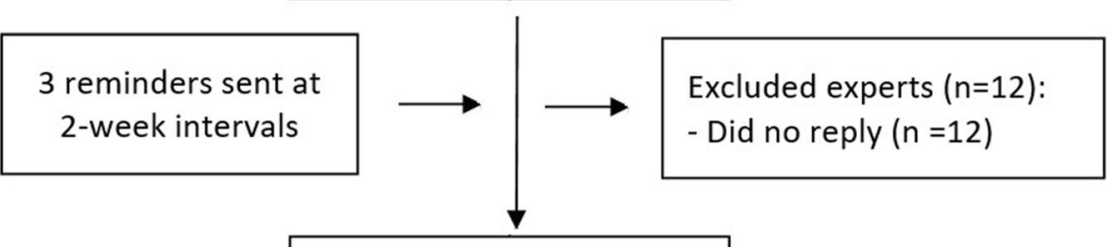

Delphi Round 3

$(n=67)$ participants who responded to the 3 rounds and those who answered the 1st or 2 first rounds. The 3-round questionnaires are available in Online Appendices 1,2 and 3, respectively.

Among all the patient-related factors, only severe comorbidity, defined by a Charlson index $>3$ or American Society of Anesthesiologists (ASA) class $>2$, was consensually related to major surgery through all three rounds. Poor nutritional status and the need for preoperative nutritional support were considered important by $69 \%$ and $66 \%$ of the participants, during rounds 1 and 2 , respectively.

Several types of interventions were proposed as examples of 'major' and 'minor' surgery (Online Appendix 4). Vascular clampage or organ ischemia (92\%), high intraoperative blood loss (90\%), intraoperative vasopressor support (>10 mcg/min noradrenalin) (77\%), long operative time $(73 \%)$ and perioperative blood transfusion $(70 \%)$ were the parameters that reached consensus (Fig. 2). In particular, operative time $>4 \mathrm{~h}$ and blood loss $>1000 \mathrm{~mL}$ were associated with a major intervention by $68 \%$ and $86 \%$ of the experts, respectively.

Postoperatively, morbidity (94\%), mortality (91\%) and the need for intensive or intermediate care $(88 \%)$ were the factors most consensually related to major surgery, with thresholds defined at $>30 \%$ for 30 -day overall morbidity and $>2 \%$ for mortality. Postoperative systemic inflammatory response was also highly related by the experts to the magnitude of surgery $(76 \%)$. Biomarkers C-reactive protein (CRP) $(77 \%)$ and lactate $(78 \%)$ were related to metabolic stress by most participants, although there was no consensus on their precise cutoffs to define major surgery (Fig. 3). Other markers did not reach consensus, such as leukocytes, procalcitonin, albumin, fibrinogen, IL-1, IL6 and TNF-alpha.

The expert panel was asked about the surgeon's subjective perception of major surgery. A feeling of stress was reported by $40 \%$, tiredness by $39 \%$ and happiness/satisfaction by $20 \%$ of participants. A strong consensus was obtained on the necessity of surgical expertise (90\%), multidisciplinary management $(87 \%)$ and high volume 
Table 1 Demographics of ESA experts

\begin{tabular}{|c|c|}
\hline & Overall, $n=67$ \\
\hline Mean age (SD) & $59(9)$ \\
\hline Male gender & $67(100 \%)$ \\
\hline \multicolumn{2}{|l|}{ Surgical specialty $^{\mathrm{a}}$} \\
\hline Hepato-pancreato-biliary & $42(40 \%)$ \\
\hline Upper gastrointestinal & $22(21 \%)$ \\
\hline Colorectal & $19(18 \%)$ \\
\hline Transplantation & $8(7 \%)$ \\
\hline Thoracic & $4(4 \%)$ \\
\hline Endocrine surgery & $4(4 \%)$ \\
\hline Vascular & $3(3 \%)$ \\
\hline Plastic & $1(1 \%)$ \\
\hline Orthopedic & $1(1 \%)$ \\
\hline Bariatric & $1(1 \%)$ \\
\hline \multicolumn{2}{|l|}{ Years of practice } \\
\hline $0-10$ years & $2(3 \%)$ \\
\hline 10-20 years & $15(22 \%)$ \\
\hline$>20$ years & $50(75 \%)$ \\
\hline \multicolumn{2}{|l|}{ Hospital structure $^{\mathrm{a}}$} \\
\hline University hospital & $69(91 \%)$ \\
\hline Community hospital & $1(1 \%)$ \\
\hline Private practice & $6(8 \%)$ \\
\hline
\end{tabular}

${ }^{\text {a Participants could give several answers; the percentages are adapted }}$ to the total of the answers center $(84 \%)$ in order to perform major surgery; $64 \%$ of the experts stipulated that a major intervention can be taught to a trainee, and $87 \%$ stated that it cannot be performed as a day-case procedure.

A detailed list of statements that did not reach consensus in rounds 1 and 2 is available in Online Appendix 5.

\section{Discussion}

There is not a common understanding among surgeons of what major surgery means. According to the panel of experts from the ESA working group, a consensus $(>70 \%$ of agreement) was reached in the following criteria defining major surgery; significant patient comorbidity, key surgical parameters (long operative duration, organ ischemia, blood loss $>1000 \mathrm{~mL}$, high vasopressor use), postoperative metabolic stress response, 30-day morbidity $>30 \%$, mortality $>2 \%$ and the need for intermediate or intensive care. These criteria should be distilled now into a concise and easy-to-use definition in order to enable widespread use in both clinical practice and medical research.

In the present study, the only preoperative factor retained for the definition of major surgery was significant comorbidity of patients, defined as a Charlson score $>3$ or ASA class $>2$. Comorbidity is associated with worse health outcomes, more complex clinical management and

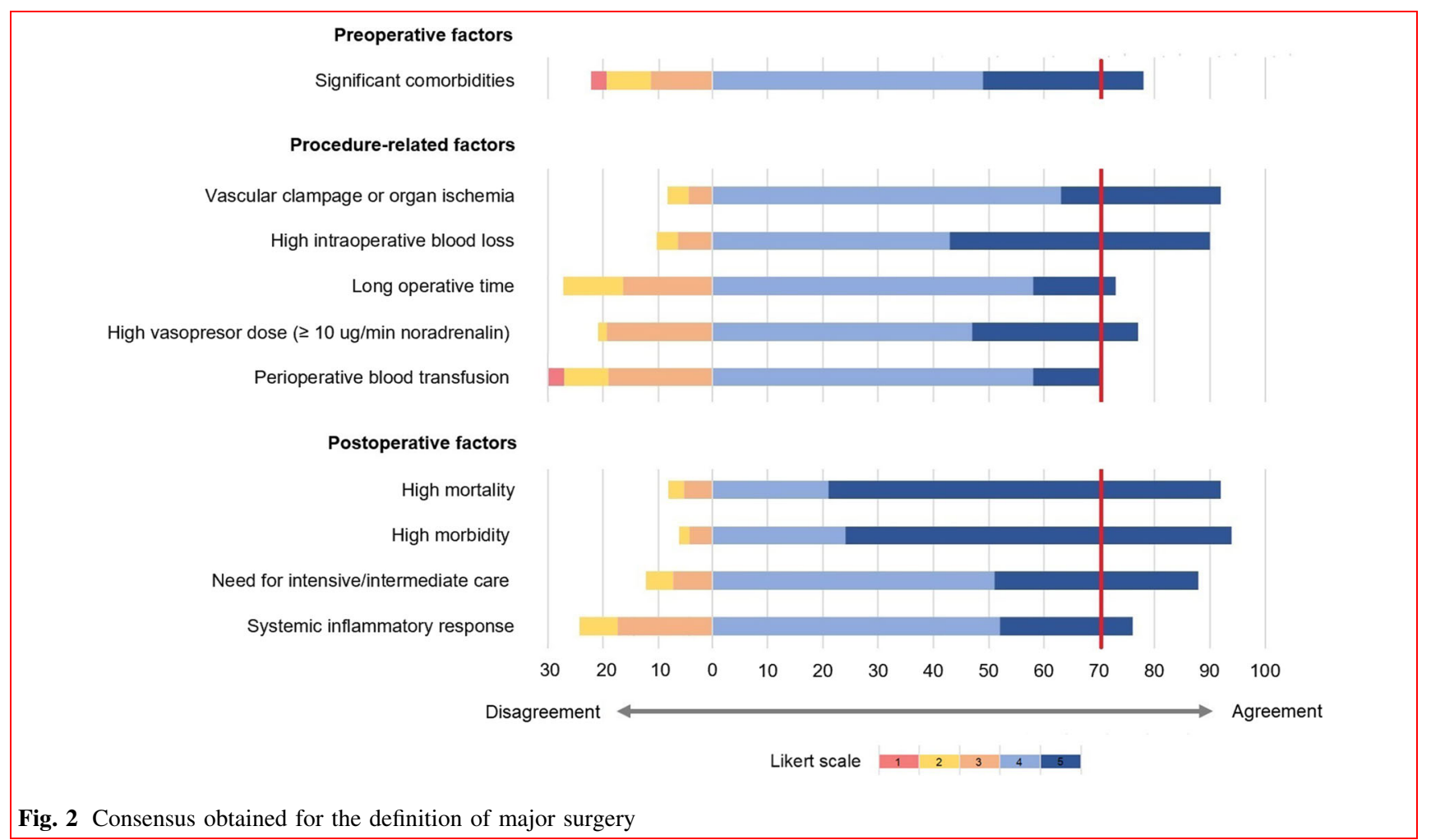




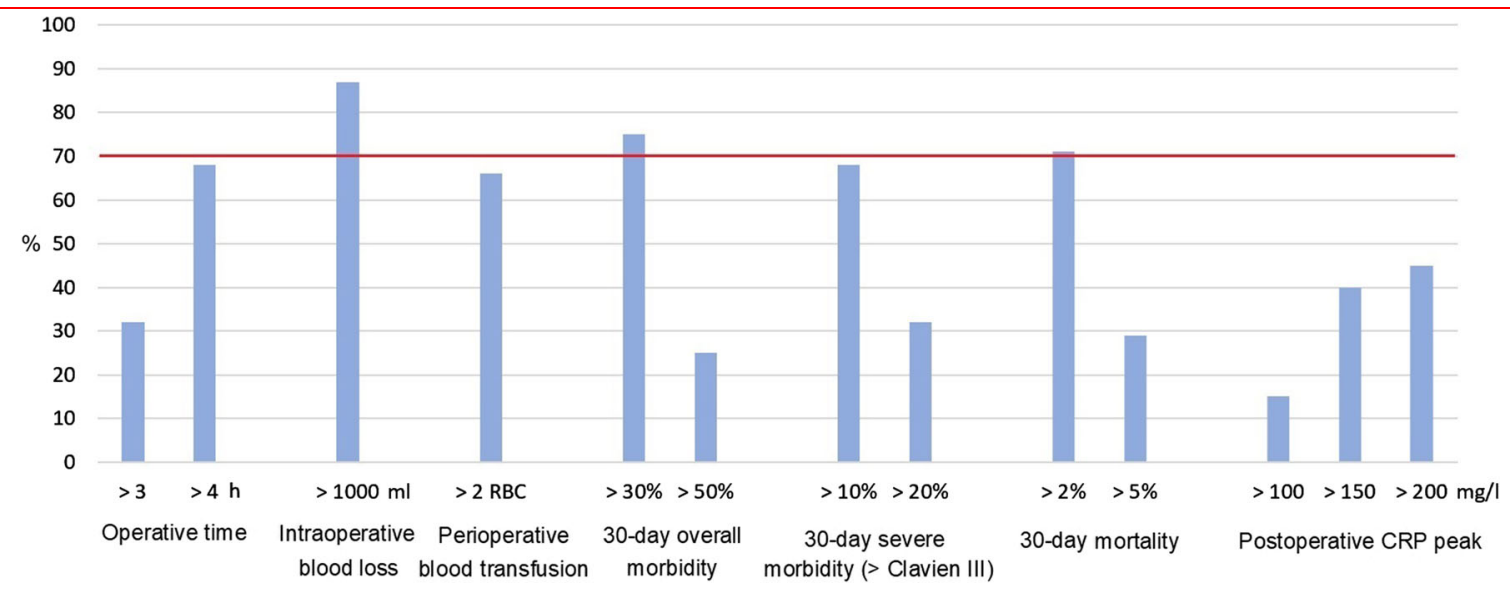

Fig. 3 Cutoffs of factors having reached consensus for the definition of major surgery

increased healthcare costs; however, there is no agreement on the meaning of the term [13]. Preexisting comorbidity was linked to increased risk of complications after major surgery in several papers published between 2009 and 2011 [14-16]. Several scoring systems have been developed to assess this risk, such as the Physiological and Operative Severity Score for the enumeration of Mortality and Morbidity (POSSUM) and the Charlson index $[9,17]$. The ASA score is widely used and easy to apply but remains prone to inter-observer variations [18, 19]. Patient age was not consensually considered as a major surgery criterion in the present study, although it has previously been described as a risk factor for morbidity and mortality after high-risk interventions [20, 21]. In a recent large-scale cohort study of 24'747 patients after major gastrointestinal surgery, overall perioperative morbidity increased twofold and mortality by 6.7 times for patients $>75$ years compared to younger patients [22]. There is thus a gap between experts' impression and previous scientific reports. A potential explanation for this fact could be that age cutoffs for 'elderly' vary significantly among the different studies between 65 and 80 years [20]. In addition, experts seemed to consider operative complexity and surgery-related characteristics as the principal determinant of major surgery, while opinions diverged as to the impact of specific patient-related factors.

Systemic inflammatory response syndrome (SIRS) is a serious condition related to systemic inflammation, organ dysfunction and organ failure [23]. It is a subset of cytokine storm that can occur after major surgery. Metabolic stress response has been extensively related to the magnitude of surgery in the literature, due to the hyperdynamic and hypermetabolic induced by surgical trauma [24]. In the present survey, systemic inflammatory response was identified as a key element to this definition. As suggested since
1975, tissue injury and ischemia-reperfusion have a systemic impact, by blunting of the cell-mediated immune system and the cascade of pro-inflammatory cytokines such as TNF- $\alpha$, IL-6, IL-8 and IL-10 [19, 25-28]. Systemic inflammation seems to be less severe or even absent during abdominal surgery without tissue ischemia [27]. What is less well studied is the biomarkers that accurately reflect surgical trauma. In a recent prospective series of patients after major abdominal surgery, a decrease in serum albumin correlated with the extent of surgery, its metabolic response and with adverse outcomes such as complications and length of stay [29]. CRP and procalcitonin have also been proposed as predictors for adverse outcomes and may be used as additional criteria of discharge $[30,31]$. In the present study, experts identified only lactate and CRP as biomarkers associated with the magnitude of surgery, without reaching a consensus on a precise cutoff. Metabolic stress after surgery is closely linked to intraoperative tissue damage and ischemia.

The expert panel identified intraoperative blood loss $>1000 \mathrm{~mL}$, hemodynamic instability, operative duration and organ ischemia as criteria to define major surgery. Previous studies have also identified severe intraoperative hemorrhage as a major risk factor for postoperative mortality [32]. Significant blood loss may trigger the cascade of inflammatory response as early as $30 \mathrm{~min}$ after the initial injury, initiating a process of multiple organ dysfunction $[33,34]$. Long operative time was also selected as a criterion for major surgery in this present survey, but no cutoff could be defined. In the literature, postoperative metabolic stress has been related to the magnitude of surgery, rather than the duration of the procedure itself [35]. Intraoperative use of high-dose vasopressors, indicating hemodynamic instability, was another item retained by the expert group, although it remains difficult to clearly define 
intraoperative instability and 'high' vasopressor doses. Data from cardiac surgery showed that vasopressor dependence was associated with postoperative renal failure, difficulty to wean from the mechanical ventilation and a longer length of stay in the intensive care unit (ICU) [36].

The attempt to identify certain types of operations as 'major' and 'minor' revealed a surprizing heterogeneity as to how experts perceive different kinds of interventions (Online Appendix 4). The experts were discrepant in about $50 \%$ of procedures. A common argument provided was that any seemingly 'minor' procedure may occasionally have major consequences, as was also previously suggested by Small et al. [2].

Postoperative morbidity $>30 \%$ and mortality $>2 \%$ were set as a threshold for major surgery by the participants. Although these cutoffs remain somewhat arbitrary, they gathered a high rate of agreement among experts from different subspecialties. Previous studies have also suggested a threshold of mortality $>1 \%$ related to major surgery [37]. Perioperative care is another element closely related to the magnitude of surgery, as multidisciplinary patient management in high-volume center has been shown to reduce short- and long-term complications in high-risk surgery [38, 39]. The present survey reinforces the importance of the expertise of both surgeon and center when endeavoring major interventions, as underlined by $>90 \%$ of experts.

One direct implication of defining an intervention as 'major' would be the level of postoperative surveillance needed. Routine increased surveillance (intermediate or intensive care) after high-risk procedures may allow for prompt recognition and management of postoperative complications, thereby limiting added morbidity and mortality [40]. A British nationwide study including more than 4 million surgical procedures showed that high-risk surgical population accounts for $12.5 \%$ of procedures but for more than $80 \%$ of deaths, and only $15 \%$ of these patients were admitted to the ICU [41]. However, it is important to consider the fact that high-dependency units are very different from country to country.

Data about surgeons' emotions are sparse in the literature, and it remains unclear what role emotions play in surgical practice [42]. In the present study, a feeling of stress, tiredness and happiness/satisfaction were reported by a minority of surgeons. The few available studies have mainly focused on feelings occurring in the context of stress, due to either an adverse event in the operating room or the occurrence of a severe postoperative complication [43, 44]. In a recent review exploring how surgeons described their practice, a gap between the myth of the surgeon (idealized image of surgeons as non-introspective, isolated, cold technicians, derived from the culture of surgeons) and their actual experience has been showed [45].

Several limitations of the present study need to be addressed. A potential selection bias could be introduced, insofar as certain parameters have been proposed by authors. However, participants had the possibility to suggest other items in free-text fields. The expert panel was a highly selected group of European academic surgeons with a majority specialized in HPB surgery, which could limit the ability to extrapolate the proposed definition to other disciplines. Intraoperative blood loss, hemodynamic instability and organ ischemia as criteria to define major surgery may be applied for major hepatic surgery, but not necessary for other types of surgery, such as rectal resection for example. Moreover, other specialists have assessed criteria of other specialties, without their competence in the field being evaluated. Furthermore, it is difficult to assess how representative the answers are, to the extent that the opinion and demographic characteristics of the 238 experts who did not answer are not known. In addition, as this survey included mostly academic surgeons (91\%), some findings may not be applicable to smaller structures, such as peripheral hospitals or private hospitals. The response rate was unfortunately low $(22 \%)$ to provide a robust extrapolation of the results to the general surgical population. The target surgeon group was chosen to include expert and experienced surgeons, aiming to provide a well-founded basis for major surgery definition. Although further validation of these results is warranted in different surgeon populations and subspecialties, this study addresses the question in a methodologically robust and clinically relevant manner. A baseline definition of what expert surgeons consider as a 'major' intervention seems to exist, and precisely the most interesting aspect of this definition is its generalizability in different types of health systems and surgery type. This preliminary proposal of a highly expert group could serve as a basis for further elaboration of this widely used, but so poorly defined term of 'major' surgery. The Delphi technique is a widely used and accepted method for achieving convergence of opinion among experts within specific questions or issues. This method was designed to transform opinion into group consensus $[8,46]$. One of the primary characteristics and advantages of the Delphi process is subject anonymity which can reduce the effects of dominant individuals which often is a concern when using group-based processes used to collect and synthesize information [7].

In conclusion, ESA experts defined major surgery according to preexisting comorbidity of the patients, extent and complexity of the procedure, its pathophysiological consequences and consecutive clinical outcomes. Further assessment of these criteria needs to be obtained in order to 
validate and refine the definition for applicability in both clinical practice and medical research.

Acknowledgements Collaborators of the ESA Study Group are: Henri Bismuth (Centre Hépato-Biliaire, Assistance Publique-Hôpitaux de Paris, Hôpital Paul Brousse, Université Paris-Sud, Villejuif, France); Michael G. Sarr (Department of Surgery, Mayo Clinic, Rochester, USA); Steven M. Strasberg (Department of Surgery, Washington University School of Medicine, St. Louis, USA); Steven D. Wexner (Department of Colorectal Surgery, Cleveland Clinic Florida, USA); Mustapha Adham (Department of Surgery, Edouard Herriot Hospital, Lyon, France); Donato F. Altomare (Department of Emergency and Organ Transplantation, University Aldo Moro, Bari, Italy); Roland Andersson (Department of Surgery, Clinical Sciences Lund, Lund University and Skåne University Hospital, Lund, Sweden); Wolf Bechstein (Department of Surgery, Frankfurt University Hospital, Frankfurt, Germany); Sebastiano Biondo (Department of General and Digestive Surgery, Bellvitge University Hospital, Barcelona, Spain); Maximilian Bockhorn (Department of General, Visceral and Thoracic Surgery, University Medical Center HamburgEppendorf, Hamburg, Germany); Luigi Bonavina (Department of Surgery, IRCCS Policlinico San Donato, University of Milan, Milano, Italy); Daniel Casanova Rituerto (Department of General Surgery, Hospital Universitario Marqués de Valdecilla, Santander, Spain); Pierre-Alain Clavien (Department of Surgery and Transplantation, Swiss HPB and Transplantation Center, University Hospital Zürich, Zürich, Switzerland); Nicolo De Manzini (Department of General Surgery, University Hospital of Trieste, Trieste, Italy); George Decker (Hôpitaux Robert Schuman, HRS-Zithaklinik, Luxembourg); Cornelis HC Dejong (Department of Surgery, Maastricht University Medical Centre, Maastricht, The Netherlands); Christos Dervenis (University of Cyprus and Department of Surgical Oncology and HPB Surgery Metropolitan Hospital, Athens, Greece); Olivier Farges (Department of HPB Surgery and Liver Transplantation, Beaujon Hospital, Clichy, France); Joan Figueras (Josep Trueta Hospital, Girona, Spain); Abraham Louis Fingerhut (Department of Surgical Research, Medical University of Graz, Austria); Helmut Friess (Department of Surgery, Klinikum rechts der Isar, Technical University, Munich, Germany); Olivier Glehen (Department of Surgical Oncology, Centre Hospitalier Lyon-Sud, Lyon 1 University, Lyon, France); Michael Gnant (Department of Surgery, Comprehensive Cancer Center, Medical University Vienna and Pancreatic Cancer Unit, Vienna, Austria); Christian Gutschow (Department of Surgery and Transplantation, University Hospital Zürich, Zürich, Switzerland); Dieter Hahnloser (Department of Visceral Surgery, Lausanne University Hospital CHUV and University of Lausanne, Lausanne, Switzerland); Bertil Hamberger (Department of Molecular Medicine and Surgery, Karolinska Institutet, Stockholm, Sweden); Jacob Frans Hamming (Department of Vascular Surgery, Leiden University Medical Centre, Leiden, the Netherlands); Arnulf $\mathrm{H}$. Hölscher (Department of General, Visceral and Cancer Surgery, University of Cologne, Cologne, Germany); Jakob R. Izbicki (Department of General, Visceral and Thoracic Surgery, University Medical Center Hamburg-Eppendorf, Hamburg, Germany); Sven Jonas (Department of Hepato-, Pancreato- and Biliary Surgery, 310Klinik, Nuremberg, Germany); Aleksandar Karamarkovic (Faculty of Medicine, University Clinical Center, Clinic for Emergency Surgery, University of Belgrade, Serbia); Henrik Kehlet (Section for Surgical Pathophysiology, Rigshospitalet Copenhagen University, Copenhagen, Denmark); Ari Kalevi Leppäniemi (Department of Abdominal Surgery, Helsinki University Central Hospital, Helsinki, Finland); Jan Lerut (Department of abdominal and transplantation surgery, University Hospitals Saint Luc, Brussels, Belgium); Pål-Dag Line (Department of Transplantation Medicine, Oslo University Hospital, Oslo, Norway); J. Peter A. Lodge (Department of HPB and
Transplant Surgery, St James's University Hospital NHS Trust, Leeds, UK); Jonathan Larmonth Meakins (Department of Surgery, McGill University Health Centre, Montreal, Canada); Marco Montorsi (General and Digestive Surgery Unit, Humanitas Research Hospital and University, Rozzano, Milan, Italy); Philippe Nafteux (Department of Thoracic Surgery and Disease of the Esophagus, KUZ Gathuisberg, Leuven, Belgium); Peter Naredi (Department of Surgery, Institute of Clinical Sciences, Sahlgrenska Academy, University of Gothenburg, Gothenburg, Sweden); Attila Oláh (Surgical Department, Petz Aladár County Teaching Hospital, Györ, Hungary); Yves Panis (Department of Colorectal Surgery, Beaujon Hospital, Assistance Publique-Hôpitaux de Paris, Université Paris VII, Clichy, France); Fernando Pardo (Clínica Universitaria de Navarra, Pamplona, Spain); Rowan W. Parks (Clinical Surgery, Royal Infirmary of Edinburgh and University of Edinburgh, UK); Sergio Pedrazzoli (Clinica Chirurgica IV, University of Padua, Italy); Patrick Pessaux (Department of General, Digestive, and Endocrine Surgery, Nouvel Hôpital Civil, Université de Strasbourg, Strasbourg, France); Hugo Pinto Marques (Hepato-Biliary-Pancreatic and Transplantation Centre, Curry Cabral Hospital, CHLC, Lisbon, Portugal); Gilberto Poggioli (Department of Medical and Surgical Sciences, Sant'Orsola, Malpighi Hospital, Alma Mater Studiorum University of Bologna, Bologna, Italy); Irinel Popescu (Department of Surgery, Fundeni Clinical Institute, Bucharest, Romania); Pauli Antero Puolakkainen (Department of Surgery, University of Helsinki and Helsinki University Hospital, Helsinki, Finland); José M. Ramia Angel (Department of General and Digestive Surgery, Hospital Universitario de Guadalajara, Guadalajara, Spain); Jari Rasanen (Department of General Thoracic and Oesophageal Surgery, Heart and Lung Centre, University of Helsinki and Helsinki University Hospital, Helsinki, Finland); John Vincent Reynolds (Department of Clinical Surgery, Trinity Centre for Health Sciences, St. James's Hospital and Trinity College Dublin, Dublin, Ireland); Riccardo Rosati (Department of Surgery, San Raffaele Hospital and San Raffaele Vita-Salute University, Milan, Italy); Hans-Detlev Saeger (Department of Visceral, Thoracic and Vascular Surgery, University Hospital Carl Gustav Carus, Technical University Dresden, Germany); Stefan Schneeberger (Department of Visceral, Transplant and Thoracic Surgery, Center of Operative Medicine, Medical University of Innsbruck, Innsbruck, Austria); Paul M. Schneider (Centre for Visceral, Thoracic and Specialized Tumor Surgery, Klinik Hirslanden, Zurich, Switzerland); Kjetil Søreide (Department of Gastrointestinal Surgery, Stavanger University Hospital, Stavanger, Norway); Dirk Stippel (Department of General, Visceral, and Cancer Surgery, University of Cologne, Köln, Germany); Christian Toso (Divisions of Abdominal and Transplant surgery, Department of Surgery, Geneva University Hospitals, Geneva, Switzerland); Jean-Jacques Tuech (Department of Surgery, Rouen University Hospital, Rouen, France); Erkki Juhani Tukiainen (Department of Plastic Surgery, Helsinki University Hospital, University of Helsinki, Helsinki, Finland); Richard Van Hillegersberg (Department of Surgery, University Medical Center Utrecht, Utrecht University, Utrecht, the Netherlands); Bas Wijnhoven (Department of Surgery, Erasmus MC, University Medical Center, Rotterdam, The Netherlands); Desmond C. Winter (Department of Colorectal Surgery, St Vincent's University Hospital, Dublin, Ireland); Giovanni Zaninotto (Department of Surgery, Imperial College, London, UK).

\section{Compliance with ethical standards}

Conflict of interest The authors declare that they have no conflict of interest. 


\section{References}

1. Earl R (1917) Definition of major and minor surgery: a question and an answer. Ann Surg 65:799

2. Small RG, Witt RE (1965) Major and minor surgery. JAMA 191:180-182

3. Straatman J, Harmsen AM, Cuesta MA et al (2015) Predictive value of C-reactive protein for major complications after major abdominal surgery: a systematic review and pooled-analysis. PLoS ONE 10:e0132995

4. Sandini M, Pinotti E, Persico I et al (2017) Systematic review and meta-analysis of frailty as a predictor of morbidity and mortality after major abdominal surgery. BJS Open 1:128-137

5. Mattar SG, Alseidi AA, Jones DB et al (2013) General surgery residency inadequately prepares trainees for fellowship: results of a survey of fellowship program directors. Ann Surg 258:440-449

6. Fiore JF Jr, Bialocerkowski A, Browning L et al (2012) Criteria to determine readiness for hospital discharge following colorectal surgery: an international consensus using the Delphi technique. Dis Colon Rectum 55:416-423

7. Murphy MK, Black NA, Lamping DL et al (1998) Consensus development methods, and their use in clinical guideline development. Health Technol Assess (Winchester, England) 2:1-88

8. Jones J, Hunter D (1995) Consensus methods for medical and health services research. BMJ (Clin Res Ed) 311:376-380

9. Charlson ME, Pompei P, Ales KL et al (1987) A new method of classifying prognostic comorbidity in longitudinal studies: development and validation. J Chronic Dis 40:373-383

10. Owens WD, Felts JA, Spitznagel EL Jr (1978) ASA physical status classifications: a study of consistency of ratings. Anesthesiology 49:239-243

11. Gero D, Gie O, Hubner M et al (2017) Postoperative ileus: in search of an international consensus on definition, diagnosis, and treatment. Langenbeck's Arch Surg 402:149-158

12. Bonrath EM, Grantcharov TP (2015) Contemporary management of paraesophaegeal hernias: establishing a European expert consensus. Surg Endosc 29:2180-2195

13. Valderas JM, Starfield B, Sibbald B et al (2009) Defining comorbidity: implications for understanding health and health services. Ann Fam Med 7:357-363

14. Kennedy GD, Rajamanickam V, O'Connor ES et al (2011) Optimizing surgical care of colon cancer in the older adult population. Ann Surg 253:508-514

15. Morris EJ, Taylor EF, Thomas JD et al (2011) Thirty-day postoperative mortality after colorectal cancer surgery in England. Gut 60:806-813

16. Rossi M, Iemma D (2009) Patients with comorbidities: what shall we do to improve the outcome. Minerva Anestesiol 75:325-327

17. Copeland GP, Jones D, Walters M (1991) POSSUM: a scoring system for surgical audit. Br J Surg 78:355-360

18. Saklad M (1941) Grading of patients for surgical procedures. Anesthesiology 2:281-284

19. Banz VM, Jakob SM, Inderbitzin D (2011) Review article: improving outcome after major surgery: pathophysiological considerations. Anesth Analg 112:1147-1155

20. Riall TS, Reddy DM, Nealon WH et al (2008) The effect of age on short-term outcomes after pancreatic resection: a populationbased study. Ann Surg 248:459-467

21. Gretschel S, Estevez-Schwarz L, Hunerbein M et al (2006) Gastric cancer surgery in elderly patients. World J Surg 30:1468-1474. https://doi.org/10.1007/s00268-005-0633-5

22. Bentrem DJ, Cohen ME, Hynes DM et al (2009) Identification of specific quality improvement opportunities for the elderly undergoing gastrointestinal surgery. Arch Surg (Chicago, Ill: 1960) 144:1013-1020

23. Hayden WR (1993) Sepsis and organ failure definitions and guidelines. Crit Care Med 21:1612-1613

24. Ishikawa M, Nishioka M, Hanaki N et al (2006) Postoperative host responses in elderly patients after gastrointestinal surgery. Hepatogastroenterology 53:730-735

25. Slade MS, Simmons RL, Yunis E et al (1975) Immunodepression after major surgery in normal patients. Surgery 78:363-372

26. Haupt W, Riese J, Mehler C et al (1998) Monocyte function before and after surgical trauma. Dig Surg 15:102-104

27. Adembri C, Kastamoniti E, Bertolozzi I et al (2004) Pulmonary injury follows systemic inflammatory reaction in infrarenal aortic surgery. Crit Care Med 32:1170-1177

28. Wakefield CH, Carey PD, Foulds S et al (1993) Changes in major histocompatibility complex class II expression in monocytes and $\mathrm{T}$ cells of patients developing infection after surgery. Br J Surg 80:205-209

29. Labgaa I, Joliat GR, Kefleyesus A et al (2017) Is postoperative decrease of serum albumin an early predictor of complications after major abdominal surgery? A prospective cohort study in a European centre. BMJ Open 7:e013966

30. Facy O, Paquette B, Orry D et al (2016) Diagnostic accuracy of inflammatory markers as early predictors of infection after elective colorectal surgery: results from the IMACORS study. Ann Surg 263:961-966

31. Giaccaglia V, Salvi PF, Antonelli MS et al (2016) Procalcitonin reveals early dehiscence in colorectal surgery: the PREDICS study. Ann Surg 263:967-972

32. Irita K, Kawashima Y, Morita K et al (2005) Supplemental survey in 2003 concerning life-threatening hemorrhagic events in the operating room. Masui Jpn J Anesthesiol 54:77-86

33. Ayala A, Perrin MM, Meldrum DR et al (1990) Hemorrhage induces an increase in serum TNF which is not associated with elevated levels of endotoxin. Cytokine 2:170-174

34. Martin C, Boisson C, Haccoun M et al (1997) Patterns of cytokine evolution (tumor necrosis factor-alpha and interleukin-6) after septic shock, hemorrhagic shock, and severe trauma. Crit Care Med 25:1813-1819

35. Pape HC, Griensven MV, Hildebrand FF et al (2008) Systemic inflammatory response after extremity or truncal fracture operations. J Trauma 65:1379-1384

36. Weis F, Kilger E, Beiras-Fernandez A et al (2006) Association between vasopressor dependence and early outcome in patients after cardiac surgery. Anaesthesia 61:938-942

37. Ghaferi AA, Birkmeyer JD, Dimick JB (2009) Variation in hospital mortality associated with inpatient surgery. N Engl J Med 361:1368-1375

38. Endo I, Kumamoto T, Matsuyama R (2017) Postoperative complications and mortality: are they unavoidable? Ann Gastroenterol Surg 1:160-163

39. Dimick JB, Pronovost PJ, Cowan JA Jr et al (2003) Variation in postoperative complication rates after high-risk surgery in the United States. Surgery 134:534-540 (discussion 540-531)

40. Sobol JB, Wunsch H (2011) Triage of high-risk surgical patients for intensive care. Crit Care (London, England) 15:217

41. Pearse RM, Harrison DA, James P et al (2006) Identification and characterisation of the high-risk surgical population in the United Kingdom. Crit Care (London, England) 10:R81

42. Orri M, Revah-Levy A, Farges O (2015) Surgeons' emotional experience of their everyday practice: a qualitative study. PLoS ONE 10:e0143763 
43. Skevington SM, Langdon JE, Giddins G (2012) 'Skating on thin ice?' Consultant surgeon's contemporary experience of adverse surgical events. Psychol Health Med 17:1-16

44. Pinto A, Faiz O, Bicknell C et al (2013) Surgical complications and their implications for surgeons' well-being. $\mathrm{Br}$ J Surg 100:1748-1755

45. Orri M, Farges O, Clavien PA et al (2014) Being a surgeon-the myth and the reality: a meta-synthesis of surgeons' perspectives about factors affecting their practice and well-being. Ann Surg 260:721-728 (discussion 728-729)

46. Hasson F, Keeney S, McKenna H (2000) Research guidelines for the Delphi survey technique. J Adv Nurs 32:1008-1015

Publisher's Note Springer Nature remains neutral with regard to jurisdictional claims in published maps and institutional affiliations. 